\title{
Green's function approach to frequency analysis of thin circular plates
}

\author{
K.K. ŻUR* \\ Faculty of Management, Bialystok University of Technology, 2 Ojca Stefana Tarasiuka St., 16-001 Kleosin, Poland
}

\begin{abstract}
The free vibration analysis of homogeneous and isotropic circular thin plates by using the Green's functions is considered. The formulae for construction of the influence function for all nodal diameters are presented in a closed form. The limited independent solutions of differential Euler equations were expanded in the Neumann power series using the method of successive approximation. This approach allows to obtain the analytical frequency equations as power series rapidly convergent to exact eigenvalues for different number of nodal diameters. The first ten dimensionless frequencies for eight different natural modes of circular plates are calculated. A part of obtained results have not been presented yet in open literature for thin circular plates. The results of investigation are in good agreement with selected results obtained by other methods presented in literature.
\end{abstract}

Key words: free vibration, circular plates, Green's function.

\section{Introduction}

In recent years, lightweight plate structures have been used in many engineering applications. Components of circular plates are commonly used in aerospace industries and aviation as well as in marine and civil engineering applications. Circular plates are the most critical structural elements in high speed rotating engineering systems such as circular saws, rotors, turbine flywheels, etc. The natural frequencies of the plates have been studied extensively for more than a century, if only because the frequency of an external load matches the natural frequency of the plate, destruction may occur.

Researchers have used various methods of analysis of dynamic behavior of plates with different boundary conditions. The work of Leissa [1] is an excellent source of information about methods used for free vibration analysis of plates. The free vibration analysis has been carried out by using a variety of weighting function methods [1] such as the Ritz method, the Galerkin method or the finite element method. In many works $[1,2]$ natural frequencies of circular plates are expressed in terms of the Bessel functions. Chakraverty et al. [3, 4] have studied the free vibration analysis of plates of various geometries by using two-dimensional boundary characteristic orthogonal polynomials in the Rayleigh-Ritz method. Wu and Liu [5, 6] proposed the generalized differential quadrature rule (GDQR) to a free vibration analysis of circular thin plates. Jaroszewicz and Zoryj [7] have studied free vibration of circular thin plates of constant and linearly variable thickness using the method of partial discretization (MPD). Ebrahimi and Rastgo [8] investigated the natural vibration behavior of circular functionally graded plates with clamped edges based on classical plate theory. Yalcin et al. [9] have studied free vibration of circular plates by using differential transformation method (DTM). Zhou et al. [10] applied the Hamiltonian approach to a solution of the free vibration problem of circular and annular thin plates. Kukla and Szewczyk [11] applied the Green's functions (influence function) and Bessel functions to the solution eigenvalue problem of annular thin plates with discrete elements such as oscillators. Similarly, Sorokin and Peake [12] have studied the free vibration analysis of sandwich plates with concentrated springs and mass by using Green's functions. An area of application of Green's functions in a free vibration analysis of isotropic beams and plates with constant and variable distribution of parameters is presented in the monograph of Kukla [13]. The application of Green's functions in free axisymmetric vibration of circular thin plates with clamped edges is presented in the book of Jaroszewicz and Zoryj [14].

In the present study, Green's functions are used to obtain ten lower natural frequencies for eight different natural modes of circular plates with different boundary conditions. A formula of construction of influence functions for different modes of uniform circular thin plates is obtained in closed form. The characteristic equations for different boundary conditions and different number of nodal lines of thin circular plates are defined. The numerical results of investigation are compared with results presented in literature.

\section{Statement of the problem}

Consider an isotropic, homogeneous circular thin plate of constant thickness $h$ in cylindrical coordinate $(r, \theta, z)$ with the $\mathrm{z}$-axis along the longitudinal direction. Geometry and coordinate system of considering plate as shown in Fig. 1. The partial differential equation for free vibration of thin plates has following form

$$
\nabla^{4} W+\frac{\rho h}{D} \frac{\partial^{2} W}{\partial t^{2}}=0
$$

\footnotetext{
*e-mail: k.zur@pb.edu.pl
} 
where $\rho$ is the mass density, $D=E h^{3} / 12\left(1-\nu^{2}\right)$ is the flexural rigidity, $E$ is Young's modulus, $\nu$ is the Poisson ratio, $\nabla^{2}=\frac{\partial^{2}}{\partial r^{2}}+\frac{1}{r} \frac{\partial}{\partial r}+\frac{1}{r^{2}} \frac{\partial^{2}}{\partial \theta^{2}}$ is the Laplacian and $W(r, \theta, t)$ is the small deflection compared with the thickness $h$ of plate.

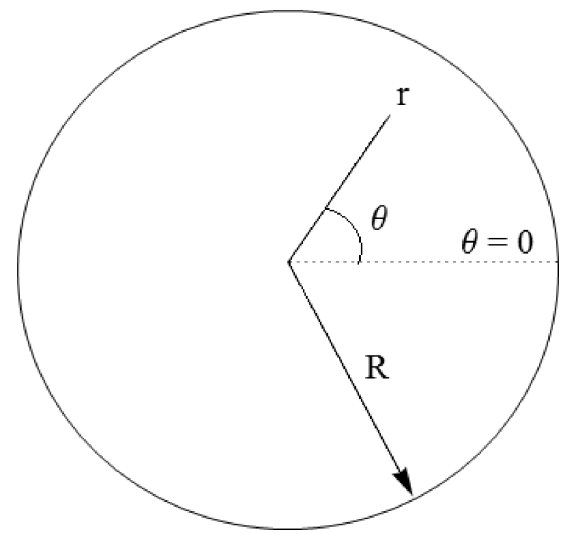

Fig. 1. Geometry and coordinate system of the circular plate

The deflection of a circular plate may be expressed as follows

$$
W(r, \theta, t)=w(r) \cos (n \theta) e^{i \omega t},
$$

where $n$ is the integer number of diagonal nodal lines, $w(r)$ is the radial mode function, $\omega$ is natural frequency, and $i^{2}=-1$. Substituting Eq. (2) into Eq. (1) using the dimensionless coordinate $\xi=r / R$ the governing differential equation of the circular plate becomes:

$$
L(w)-\lambda^{2} w=0,
$$

where

$$
\begin{gathered}
L(w) \equiv \frac{d^{4} w}{d \xi^{4}}+\frac{2}{\xi} \frac{d^{3} w}{d \xi^{3}}-\frac{\left(1+2 n^{2}\right)}{\xi^{2}} \frac{d^{2} w}{d \xi^{2}} \\
+\frac{\left(1+2 n^{2}\right)}{\xi^{3}} \frac{d w}{d \xi}+\frac{\left(n^{4}-4 n^{2}\right)}{\xi^{4}} w
\end{gathered}
$$

is differential operator and

$$
\lambda=\omega R^{2} \sqrt{\rho h / D}
$$

is dimensionless frequency of vibration.

The boundary conditions at the outer edge $(\xi=1)$ of the circular plate may be one of the following: clamped, simply supported, free, sliding supports and elastic supports. These conditions may be written in terms of the radial mode function $w(\xi)$ in the following form:

Clamped:

$$
\begin{aligned}
& \left.w(\xi)\right|_{\xi=1}=0, \\
& \left.\frac{d w}{d \xi}\right|_{\xi=1}=0 .
\end{aligned}
$$

Simple supports:

$$
\begin{gathered}
\left.w(\xi)\right|_{\xi=1}=0 \\
\left.M(w)\right|_{\xi=1}=\left[\frac{d^{2} w}{d \xi^{2}}+\frac{\nu}{\xi} \frac{d w}{d \xi}-\frac{\nu n^{2}}{\xi^{2}} w\right]_{\xi=1}=0 .
\end{gathered}
$$

Free:

$$
\begin{aligned}
&\left.M(w)\right|_{\xi=1}=0 \\
&\left.V(w)\right|_{\xi=1}= {\left[\frac{d^{3} w}{d \xi^{3}}+\frac{1}{\xi} \frac{d^{2} w}{d \xi^{2}}-\left(\frac{1+2 n^{2}-\nu n^{2}}{\xi^{2}}\right) \frac{d w}{d \xi}\right.} \\
&\left.+\left(\frac{3 n^{2}-\nu n^{2}}{\xi^{3}}\right) w\right]_{\xi=1}=0 .
\end{aligned}
$$

Sliding supports:

$$
\begin{gathered}
\left.\frac{d w}{d \xi}\right|_{\xi=1}=0, \\
\left.V(w)\right|_{\xi=1}=0 .
\end{gathered}
$$

Elastic supports:

$$
\begin{gathered}
\left.\Phi(w)\right|_{\xi=1}=\left[\left(\frac{d^{2} w}{d \xi^{2}}+\nu \frac{d w}{d \xi}-\frac{\nu n^{2}}{\xi^{2}} w\right)+\phi \frac{d w}{d \xi}\right]_{\xi=1}=0 \\
\left.\Psi(w)\right|_{\xi=1}=\left[\left(\frac{d^{3} w}{d \xi^{3}}+\frac{d^{2} w}{d \xi^{2}}+\left(\nu n^{2}-2 n^{2}-1\right)\right.\right. \\
\left.\left.\cdot \frac{d w}{d \xi}+\left(3 n^{2}-\nu n^{2}\right) w\right)-\psi w\right]_{\xi=1}=0 .
\end{gathered}
$$

$M(w)$ and $V(w)$ are the normalized radial bending moment and the normalized effective shear force, respectively. $\phi=K_{\phi} R / D$ and $\psi=K_{\psi} R^{3} / D$ are normalized parameters of elastic supports. $K_{\phi}$ and $K_{\psi}$ are rotational and translational spring constants (Fig. 2), respectively.

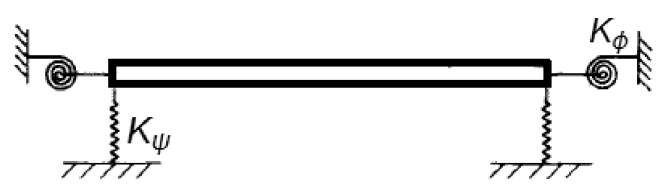

Fig. 2. The cross-section of uniform circular plate with elastic supports

\section{Mathematical background}

The formula of construction of the Green's function (general solution) for homogeneous differential equations

$$
L(y) \equiv \sum_{k=0}^{n} p_{k}(x) \frac{d^{k} y}{d x^{k}}=0, \quad a<x<b
$$

has following form

$$
K(x, \alpha)=\sum_{k=1}^{n} C_{k}^{\prime} y_{k}(x),
$$

where coefficients $p_{k}(x)$ are continuous functions, $p_{n}(x) \neq 0$ for $x \in[a b]$ and $\alpha$ is a position where all discrete elements could be mounted $[11,14]$. 
Green's function approach to frequency analysis of thin circular plates

Constants $C_{k}^{\prime}$ have form [13]

$$
\begin{array}{cccccc}
\multicolumn{7}{c}{C_{k}^{\prime}=\frac{(-1)^{n+k}}{p_{n}(\alpha) W_{n}(\alpha)}} \\
y_{1}(\alpha) & \cdots & y_{k-1}(\alpha) & y_{k+1}(\alpha) & \cdots & y_{n}(\alpha) \\
y_{1}^{\prime}(\alpha) & \cdots & y_{k-1}^{\prime}(\alpha) & y_{k+1}^{\prime}(\alpha) & \cdots & y_{n}^{\prime}(\alpha) \\
\vdots & \cdots & \vdots & \vdots & \vdots & \vdots \\
y_{1}^{(n-2)}(\alpha) & \cdots & y_{k-1}^{(n-2)}(\alpha) & y_{k+1}^{(n-2)}(\alpha) & \cdots & y_{n}^{(n-2)}(\alpha)
\end{array} \mid
$$

where $W_{n}$ is Wronskian

$$
W_{n}(x)=\left|y_{k}^{(i-1)}(x)\right|_{1 \leq i, k \leq n}
$$

and $y_{k}(x)$ are linear independent solutions of Eq. (1), then Wronskian satisfies condition $W_{n}(\alpha) \neq 0$.

The other formula of construction of the Green's function for homogeneous differential equations has form [14]

$$
K(x, \alpha)=\frac{|A|}{W(\alpha) p_{n}(\alpha)}
$$

where

$$
\begin{gathered}
|A|=\left|\begin{array}{cccc}
y_{1}(\alpha) & y_{2}(\alpha) & \ldots & y_{n}(\alpha) \\
y_{1}^{\prime}(\alpha) & y_{2}^{\prime}(\alpha) & \ldots & y_{n}^{\prime}(\alpha) \\
\vdots & \vdots & \ldots & \vdots \\
y_{1}^{(n-2)}(\alpha) & y_{2}^{(n-2)}(\alpha) & \ldots & y_{n}^{(n-2)}(\alpha) \\
y_{1}(x) & y_{2}(x) & \ldots & y_{n}(x)
\end{array}\right|, \\
W(\alpha)=\left|\begin{array}{cccc}
y_{1}(\alpha) & y_{2}(\alpha) & \ldots & y_{n}(\alpha) \\
y_{1}^{\prime}(\alpha) & y_{2}^{\prime}(\alpha) & \ldots & y_{n}^{\prime}(\alpha) \\
\vdots & \vdots & \vdots & \vdots \\
y_{1}^{(n-1)}(\alpha) & y_{2}^{(n-1)}(\alpha) & \ldots & y_{n}^{(n-1)}(\alpha)
\end{array}\right| .
\end{gathered}
$$

Additionally, the functions $K(x, \alpha)$ must satisfy conditions presented in the following form [13]

$$
\begin{gathered}
\left.\frac{\partial^{i} K(x, \alpha)}{\partial x^{i}}\right|_{x=\alpha}=0, \quad i=0,1, \ldots, n-2, \\
\left.\frac{\partial^{n-1} K(x, \alpha)}{\partial x^{n-1}}\right|_{x=\alpha}=\frac{1}{p_{n}(\alpha)} .
\end{gathered}
$$

\section{Green's function}

The characteristic equation of homogeneous differential Euler equation

$$
\begin{gathered}
L(w) \equiv \frac{d^{4} w}{d \xi^{4}}+\frac{2}{\xi} \frac{d^{3} w}{d \xi^{3}}-\frac{\left(1+2 n^{2}\right)}{\xi^{2}} \frac{d^{2} w}{d \xi^{2}} \\
+\frac{\left(1+2 n^{2}\right)}{\xi^{3}} \frac{d w}{d \xi}+\frac{\left(n^{4}-4 n^{2}\right)}{\xi^{4}} w=0
\end{gathered}
$$

has following form

$$
s^{4}-4 s^{3}+\left(4-2 n^{2}\right) s^{2}+4 n^{2} s-n^{2}\left(4-n^{2}\right)=0 .
$$

The roots of Eq. (20) have form

$$
s_{1}=n, \quad s_{2}=-n, \quad s_{3}=2-n, \quad s_{4}=2+n .
$$

The linear independent solutions of Eq. (15) are

$$
\begin{gathered}
w_{1}(\xi)=\xi^{n}, \quad w_{2}(\xi)=\xi^{-n}, \\
w_{3}(\xi)=\xi^{2-n}, \quad w_{4}(\xi)=\xi^{2+n} .
\end{gathered}
$$

The Green's function (solution of homogeneous Euler equation $L(K(\xi, \alpha))=0$ ) for different nodal diameters may be received from Eq. (15) presented in the following form

$$
K_{n}(\xi, \alpha)=\frac{\left|A_{n}\right|}{W(\alpha)_{n} p_{n}(\alpha)},
$$

where $p_{n}(\alpha)=1$ is coefficient placed on before highest order of derivative of Euler differential Eq. (19) and

$\left|A_{n}\right|=\left|\begin{array}{cccc}\alpha^{n} & \alpha^{-n} & \alpha^{2-n} & \alpha^{2+n} \\ \frac{d\left(\alpha^{n}\right)}{d \alpha} & \frac{d\left(\alpha^{-n}\right)}{d \alpha} & \frac{d\left(\alpha^{2-n}\right)}{d \alpha} & \frac{d\left(\alpha^{2+n}\right)}{d \alpha} \\ \frac{d^{2}\left(\alpha^{n}\right)}{d \alpha^{2}} & \frac{d^{2}\left(\alpha^{-n}\right)}{d \alpha^{2}} & \frac{d^{2}\left(\alpha^{2-n}\right)}{d \alpha^{2}} & \frac{d^{2}\left(\alpha^{2+n}\right)}{d \alpha^{2}} \\ \xi^{n} & \xi^{-n} & \xi^{2-n} & \xi^{2+n}\end{array}\right|$

$$
W(\alpha)_{n}=\left|\begin{array}{cccc}
\alpha^{n} & \alpha^{-n} & \alpha^{2-n} & \alpha^{2+n} \\
\frac{d\left(\alpha^{n}\right)}{d \alpha} & \frac{d\left(\alpha^{-n}\right)}{d \alpha} & \frac{d\left(\alpha^{2-n}\right)}{d \alpha} & \frac{d\left(\alpha^{2+n}\right)}{d \alpha} \\
\frac{d^{2}\left(\alpha^{n}\right)}{d \alpha^{2}} & \frac{d^{2}\left(\alpha^{-n}\right)}{d \alpha^{2}} & \frac{d^{2}\left(\alpha^{2-n}\right)}{d \alpha^{2}} & \frac{d^{2}\left(\alpha^{2+n}\right)}{d \alpha^{2}} \\
\frac{d^{3}\left(\alpha^{n}\right)}{d \alpha^{3}} & \frac{d^{3}\left(\alpha^{-n}\right)}{d \alpha^{3}} & \frac{d^{3}\left(\alpha^{2-n}\right)}{d \alpha^{3}} & \frac{d^{3}\left(\alpha^{2+n}\right)}{d \alpha^{3}}
\end{array}\right|,
$$

After calculations, the Green's function (GF) for different number nodal diameters $n$ has following form

$$
\begin{aligned}
& K_{n}(\xi, \alpha)=\frac{\alpha^{n+1} \xi^{-n+2}-\xi^{n} \alpha^{-n+3}}{8 n^{2}-8 n} \\
& +\frac{\alpha^{-n+1} \xi^{n+2}-\xi^{-n} \alpha^{n+3}}{8 n^{2}+8 n}, \quad n \geq 2
\end{aligned}
$$

and satisfies conditions from Eq. (18)

$$
\begin{gathered}
K_{n}(\alpha, \alpha)=\left.\frac{\partial K_{n}(\xi, \alpha)}{\partial \xi}\right|_{\xi=\alpha} \\
=\left.\frac{\partial^{2} K_{n}(\xi, \alpha)}{\partial \xi^{2}}\right|_{\xi=\alpha}=0, \\
\left.\frac{\partial^{3} K_{n}(\xi, \alpha)}{\partial \xi^{3}}\right|_{\xi=\alpha}=1 .
\end{gathered}
$$

The function $K_{n}(\xi, \alpha)$ is indeterminate for $n=0$ and $n=1$, then the Green's functions for this values have following form

$$
\begin{gathered}
\lim _{n \rightarrow 0} K_{n}(\xi, \alpha)=\frac{\alpha}{4}\left[\alpha^{2}-\xi^{2}+\left(\xi^{2}+\alpha^{2}\right) \ln \frac{\xi}{\alpha}\right], \\
\lim _{n \rightarrow 1} K_{n}(\xi, \alpha)=\frac{\xi^{4}-\alpha^{4}+4 \xi^{2} \alpha^{2} \ln \frac{\alpha}{\xi}}{16 \xi} .
\end{gathered}
$$


In Table 1 the examples of formulas of Green's function for seven nodal diameters are presented.

Table 1

The formulas of Green's functions for the different number of nodal diameters

\begin{tabular}{ll}
\hline \hline$n$ & $K_{0}(\xi, \alpha)=\frac{\alpha}{4}\left[\alpha^{2}-\xi^{2}+\left(\xi^{2}+\alpha^{2}\right) \ln \frac{\xi}{\alpha}\right]$ \\
\hline 1 & $K_{1}(\xi, \alpha)=\frac{\xi^{4}-\alpha^{4}+4 \xi^{2} \alpha^{2} \ln \frac{\alpha}{\xi}}{16 \xi}$ \\
\hline 3 & $K_{2}(\xi, \alpha)=\frac{\left(\xi^{2}-\alpha^{2}\right)^{3}}{48 \xi^{2} \alpha}$ \\
\hline 4 & $K_{3}(\xi, \alpha)=\frac{\left(\xi^{2}-\alpha^{2}\right)^{3}\left(\xi^{2}+\alpha^{2}\right)}{96 \xi^{3} \alpha^{2}}$ \\
\hline 5 & $K_{5}(\xi, \alpha)=\frac{1}{160}\left(\frac{\alpha^{5}}{\xi^{2}}-\frac{\xi^{4}}{\alpha}\right)+\frac{1}{160}\left(\frac{\xi^{6}}{\alpha^{3}}-\frac{\alpha^{7}}{\xi^{4}}\right)$ \\
\hline 6 & $K_{6}(\xi, \alpha)=\frac{1}{240}\left(\frac{\alpha^{7}}{\xi^{4}}-\frac{\xi^{6}}{\alpha^{3}}\right)+\frac{1}{240}\left(\frac{\xi^{7}}{\alpha^{4}}-\frac{\alpha^{8}}{\xi^{5}}\right)$ \\
\hline
\end{tabular}

\section{Solution}

The ordinary differential equations with constant or variable coefficients can be transformed to the Fredholm or the Volterra integral equations using e.g. Fubini's method [17]. The solutions of this equations are solutions of the transformed ordinary differential equation. If the Green's function (kernel of integral equation) is well known, the linear independent solutions can be expanded in the Neumann power series rapidly convergent to eigenvalues (spectrum of integral kernel) based on the method of successive approximations [18].

The limited (for $\xi=0$ ) independent solutions of Eq. (19) are $w_{1}(\xi)=\xi^{n}$ and $w_{2}(\xi)=\xi^{n+2}$. This solutions were expanded in the Neumann power series in the following form

$$
\begin{gathered}
K_{n}(\xi, \lambda)_{u}=\xi^{n}+\sum_{i=1}^{\eta} K_{i}(\xi)_{u} \cdot \lambda^{2 i}, \quad \lambda \in R^{+} \\
K_{n}(\xi, \lambda)_{v}=\xi^{n+2}+\sum_{i=1}^{\eta} K_{i}(\xi)_{v} \cdot \lambda^{2 i}
\end{gathered}
$$

where $K_{i}(\xi)_{u}$ and $K_{i}(\xi)_{v}$ are iterated kernels [18] presented in the following form

$$
\begin{aligned}
K_{i}(\xi)_{u} & =\int_{0}^{\xi} K_{n}(\xi, \alpha) K_{i-1}(\alpha)_{u} d \alpha \\
K_{i}(\xi)_{v} & =\int_{0}^{\xi} K_{n}(\xi, \alpha) K_{i-1}(\alpha)_{v} d \alpha
\end{aligned}
$$

and $\eta$ is the degree of approximations.
$K_{0}(\alpha)_{u}$ and $K_{0}(\alpha)_{v}$ are the limited independent solutions depending on number of nodal lines presented in the following form

$$
\begin{gathered}
K_{0}(\alpha)_{u}=\alpha^{n}, \\
K_{0}(\alpha)_{v}=\alpha^{n+2} .
\end{gathered}
$$

The characteristic equations $\Delta_{n}=0$ for different boundary conditions and different values of parameter $n$ are obtained from well known the characteristic determinants given by: Clamped:

$$
\Delta_{n}(\lambda) \equiv\left|\begin{array}{cc}
K_{n}(\xi, \lambda)_{u} & K_{n}(\xi, \lambda)_{v} \\
\frac{\partial K_{n}(\xi, \lambda)_{u}}{\partial \xi} & \frac{\partial K_{n}(\xi, \lambda)_{v}}{\partial \xi}
\end{array}\right|_{\xi=1} ;
$$

Simply supported:

$$
\Delta_{n}(\lambda) \equiv\left|\begin{array}{cc}
K_{n}(\xi, \lambda)_{u} & K_{n}(\xi, \lambda)_{v} \\
M\left[K_{n}(\xi, \lambda)_{u}\right] & M\left[K_{n}(\xi, \lambda)_{v}\right]
\end{array}\right|_{\xi=1} ;
$$

Free:

$$
\Delta_{n}(\lambda) \equiv\left|\begin{array}{cc}
M\left[K_{n}(\xi, \lambda)_{u}\right] & M\left[K_{n}(\xi, \lambda)_{v}\right] \\
V\left[K_{n}(\xi, \lambda)_{u}\right] & V\left[K_{n}(\xi, \lambda)_{v}\right]
\end{array}\right|_{\xi=1} ;
$$

Sliding supports:

$$
\Delta_{n}(\lambda) \equiv\left|\begin{array}{cc}
\frac{\partial K_{n}(\xi, \lambda)_{u}}{\partial \xi} & \frac{\partial K_{n}(\xi, \lambda)_{v}}{\partial \xi} \\
V\left[K_{n}(\xi, \lambda)_{u}\right] & V\left[K_{n}(\xi, \lambda)_{v}\right]
\end{array}\right|_{\xi=1} ;
$$

Elastic supports:

$$
\Delta_{n}(\lambda) \equiv\left|\begin{array}{ll}
\Phi\left[K_{n}(\xi, \lambda)_{u}\right] & \Phi\left[K_{n}(\xi, \lambda)_{v}\right] \\
\Psi\left[K_{n}(\xi, \lambda)_{u}\right] & \Psi\left[K_{n}(\xi, \lambda)_{v}\right]
\end{array}\right|_{\xi=1} .
$$

For all boundary conditions formula of $\Delta_{n}$ has following form

$$
\Delta_{n}=a_{0}+\sum_{i=1}^{\eta}(-1)^{i} a_{i} \lambda^{2 i}
$$

where $a_{0}, a_{1}, \ldots, a_{\eta}$ are coefficients of characteristic equations depending on boundary conditions and number of nodal lines $n$.

\section{Numerical results}

The numerical results for the first ten dimensionless frequencies for different boundary conditions are presented in Tables $2-8$. The nodal diameters are considered for the values between 0 and 7. The Neumann power series Eq. (30) were expanded only for $\eta=25$. Poisson ratio is taken as $\nu=0.3$ for all considered cases. The numerical results for free, clamped and simply supported circular plates are presented in Tables 2-4 with comparison to Refs. [1, 6, 9, 10, 16]. The numerical results for plates with sliding supports are shown in Table 5 with comparison to Refs. [6, 15]. The eigenvalues for plates with elastic supports and different values of elastic parameters on the edges are presented in Tables 6-8 with comparison to Refs. [6, 15]. 
Green's function approach to frequency analysis of thin circular plates

Table 2

The first ten lower dimensionless frequencies $\lambda$ of free vibration of clamped circular plates

\begin{tabular}{|c|c|c|c|c|c|c|c|c|c|}
\hline \multirow{2}{*}{\multicolumn{2}{|c|}{$\begin{array}{c}\text { Dimensionless } \\
\text { frequency, } \lambda\end{array}$}} & \multicolumn{8}{|c|}{ The number of nodal diameters, $n$} \\
\hline & & 0 & 1 & 2 & 3 & 4 & 5 & 6 & 7 \\
\hline \multirow[t]{5}{*}{$\lambda_{0}$} & GF & 10.216 & 21.260 & 34.877 & 51.030 & 69.665 & 90.739 & 114.213 & 140.056 \\
\hline & Ref. [1] & 10.215 & 21.260 & 34.88 & 51.04 & 69.665 & 90.739 & 114.212 & 140.056 \\
\hline & Ref. [6] & 10.216 & 21.260 & 34.877 & 51.030 & 69.666 & 90.739 & - & - \\
\hline & Ref. [9] & 10.215 & 21.260 & 34.877 & 51.03 & 69.665 & 90.738 & - & - \\
\hline & Ref. [16] & 10.215 & 21.260 & 34.877 & - & - & - & - & - \\
\hline \multirow[t]{5}{*}{$\lambda_{1}$} & GF & 39.771 & 60.828 & 84.582 & 111.021 & 140.108 & 171.803 & 206.071 & 242.878 \\
\hline & Ref. [1] & 39.771 & 60.82 & 84.58 & 111.01 & 140.107 & 171.802 & 206.070 & 242.878 \\
\hline & Ref. [6] & 39.771 & 60.829 & 84.583 & 111.021 & 140.108 & 171.803 & - & - \\
\hline & Ref. [9] & 39.771 & 60.828 & 84.582 & 111.021 & 140.107 & 171.802 & - & - \\
\hline & Ref. [16] & 39.771 & 60.828 & 84.582 & - & - & - & - & - \\
\hline \multirow[t]{5}{*}{$\lambda_{2}$} & GF & 89.104 & 120.079 & 153.815 & 190.304 & 229.519 & 271.428 & 316.002 & 363.210 \\
\hline & Ref. [1] & 89.104 & 120.08 & 153.81 & 190.30 & 229.518 & 271.428 & 316.001 & 363.209 \\
\hline & Ref. [6] & 89.104 & 120.079 & 153.815 & 190.304 & 229.519 & 271.428 & - & - \\
\hline & Ref. [9] & 89.104 & 120.079 & 153.815 & 190.303 & 229.518 & 271.428 & - & - \\
\hline & Ref. [16] & 89.104 & 120.079 & 153.815 & - & - & - & - & - \\
\hline \multirow[t]{5}{*}{$\lambda_{3}$} & GF & 158.184 & 199.053 & 242.721 & 289.180 & 338.411 & 390.389 & 445.089 & 502.483 \\
\hline & Ref. [1] & 158.183 & 199.06 & 242.71 & 289.17 & 338.411 & 390.389 & - & - \\
\hline & Ref. [6] & 158.184 & 199.053 & 242.721 & 289.180 & 338.411 & 390.389 & - & - \\
\hline & Ref. [9] & 158.184 & 199.053 & 242.720 & 289.179 & 338.411 & 390.389 & - & - \\
\hline & Ref. [16] & 158.184 & 199.053 & 242.721 & - & - & - & - & - \\
\hline \multirow[t]{5}{*}{$\lambda_{4}$} & $\mathrm{GF}$ & 247.006 & 297.760 & 351.336 & 407.730 & 466.925 & 528.902 & 593.639 & 661.112 \\
\hline & Ref. [1] & 247.005 & 297.77 & 351.38 & 407.72 & - & - & - & - \\
\hline & Ref. [6] & 247.006 & 297.760 & 351.336 & 407.730 & 466.925 & 528.902 & - & - \\
\hline & Ref. [9] & 247.006 & 297.76 & 351.335 & 407.729 & 466.925 & 528.902 & - & - \\
\hline & Ref. [16] & 247.007 & 297.761 & 351.337 & - & - & - & - & - \\
\hline \multirow[t]{3}{*}{$\lambda_{5}$} & GF & 355.569 & 416.203 & 479.675 & 545.983 & 615.114 & 687.051 & 761.776 & 839.268 \\
\hline & Ref. [1] & 355.568 & 416.20 & 479.65 & 545.97 & - & - & - & - \\
\hline & Ref. [6] & 355.569 & 416.203 & 479.675 & 545.983 & 615.114 & 687.051 & - & - \\
\hline \multirow{2}{*}{$\lambda_{6}$} & GF & 483.872 & 554.382 & 627.744 & 703.955 & 783.004 & 864.877 & 949.558 & 1037.03 \\
\hline & Ref. [1] & 483.872 & 554.37 & 627.75 & 703.95 & - & - & - & - \\
\hline \multirow[t]{2}{*}{$\lambda_{7}$} & GF & 631.915 & 712.300 & 795.546 & 881.652 & 970.607 & 1062.40 & 1157.02 & 1254.45 \\
\hline & Ref. [1] & 631.914 & 712.30 & 795.52 & 881.67 & - & - & - & - \\
\hline \multirow[t]{2}{*}{$\lambda_{8}$} & GF & 799.697 & 889.956 & 983.084 & 1079.08 & 1177.93 & 1279.64 & 1384.18 & 1491.55 \\
\hline & Ref. [1] & 799.762 & 889.95 & 983.07 & 1079.0 & - & - & - & - \\
\hline \multirow[t]{2}{*}{$\lambda_{9}$} & GF & 987.219 & 1087.35 & 1190.36 & 1296.24 & 1404.98 & 1516.52 & 1629.73 & 1734.10 \\
\hline & Ref. [1] & 987.216 & 1087.4 & 1190.4 & 1296.2 & - & - & - & - \\
\hline
\end{tabular}

Table 3

The first ten lower dimensionless frequencies $\lambda$ of free vibration of free circular plates

\begin{tabular}{|c|c|c|c|c|c|c|c|c|c|}
\hline \multirow{2}{*}{\multicolumn{2}{|c|}{$\begin{array}{c}\text { Dimensionless } \\
\text { frequency, } \lambda\end{array}$}} & \multicolumn{8}{|c|}{ The number of nodal diameters, $n$} \\
\hline & & 0 & 1 & 2 & 3 & 4 & 5 & 6 & 7 \\
\hline \multirow[t]{5}{*}{$\lambda_{0}$} & GF & 9.003 & 20.474 & 5.358 & 12.439 & 21.835 & 33.494 & 47.378 & 63.455 \\
\hline & Ref. [1] & 9.084 & 20.52 & 5.253 & 12.23 & 21.6 & 33.1 & 46.2 & - \\
\hline & Ref. [6] & 9.003 & 20.475 & 5.358 & 12.439 & 21.835 & 33.495 & - & - \\
\hline & Ref. [9] & 9.003 & 20.474 & 5.358 & 12.439 & 21.835 & 33.494 & - & - \\
\hline & Ref. [16] & 9.003 & 20.474 & 5.358 & - & - & - & - & - \\
\hline \multirow[t]{5}{*}{$\lambda_{1}$} & GF & 38.443 & 59.811 & 35.260 & 53.007 & 73.542 & 96.755 & 122.57 & 150.928 \\
\hline & Ref. [1] & 38.55 & 59.86 & 35.25 & 52.91 & 73.1 & 95.8 & 121.0 & - \\
\hline & Ref. [6] & 38.443 & 59.812 & 35.260 & 53.008 & 73.543 & 96.755 & - & - \\
\hline & Ref. [9] & 38.443 & 59.811 & 35.260 & 53.007 & 73.542 & 96.755 & - & - \\
\hline & Ref. [16] & 38.443 & 59.812 & 35.260 & - & - & - & - & - \\
\hline \multirow[t]{5}{*}{$\lambda_{2}$} & GF & 87.750 & 118.957 & 84.366 & 111.945 & 142.431 & 175.735 & 211.789 & 250.535 \\
\hline & Ref. [1] & 87.80 & 119.0 & 83.9 & 111.3 & 142.8 & 175.0 & 210.3 & - \\
\hline & Ref. [6] & 87.750 & 118.957 & 84.366 & 111.945 & 142.431 & 175.735 & - & - \\
\hline & Ref. [9] & 87.750 & 118.957 & 84.366 & 111.945 & 142.431 & 175.735 & - & - \\
\hline & Ref. [16] & 87.753 & 118.961 & 84.361 & - & - & - & - & - \\
\hline \multirow{5}{*}{$\lambda_{3}$} & GF & 156.818 & 197.872 & 153.306 & 190.692 & 231.03 & 274.252 & 320.299 & 369.121 \\
\hline & Ref. [1] & 157.0 & 198.2 & 154.0 & 192.1 & 232.3 & 274.6 & 319.7 & - \\
\hline & Ref. [6] & 156.818 & 197.872 & 153.306 & 190.692 & 231.03 & 274.252 & - & - \\
\hline & Ref. [9] & 156.818 & 197.871 & 153.306 & 190.692 & 231.03 & 274.252 & - & - \\
\hline & Ref. [16] & 156.826 & 197.883 & 153.310 & - & - & - & - & - \\
\hline \multirow[t]{5}{*}{$\lambda_{4}$} & GF & 245.634 & 296.54 & 242.036 & 289.238 & 339.413 & 392.505 & 448.467 & 507.254 \\
\hline & Ref. [1] & 245.9 & 296.9 & 242.7 & 290.7 & 340.4 & 392.4 & 447.3 & - \\
\hline & Ref. [6] & 245.634 & 296.540 & 242.036 & 289.238 & 339.413 & 392.505 & - & - \\
\hline & Ref. [9] & 245.633 & 296.54 & 242.036 & 289.238 & 339.413 & 392.505 & - & - \\
\hline & Ref. [16] & 245.651 & 296.564 & 242.049 & - & - & - & - & - \\
\hline \multirow[t]{3}{*}{$\lambda_{5}$} & GF & 354.192 & 414.956 & 350.534 & 407.562 & 467.573 & 530.521 & 596.365 & 665.069 \\
\hline & Ref. [1] & 354.6 & 415.3 & 350.8 & 408.4 & 467.9 & 529.5 & 593.9 & - \\
\hline & Ref. [6] & - & - & 350.534 & 407.562 & 467.573 & 530.521 & - & - \\
\hline \multirow[t]{2}{*}{$\lambda_{6}$} & GF & 482.491 & 553.115 & 478.79 & 545.651 & 615.501 & 688.3 & 764.014 & 842.609 \\
\hline & Ref. [1] & 483.1 & 553.0 & 479.2 & 546.2 & 615.0 & 686.4 & 760.1 & - \\
\hline \multirow[t]{2}{*}{$\lambda_{7}$} & GF & 630.532 & 711.017 & 626.795 & 703.497 & 783.189 & 865.835 & 951.398 & 1039.89 \\
\hline & Ref. [1] & 631.0 & 711.3 & 627.0 & 703.3 & 781.8 & 864.4 & 952.3 & - \\
\hline \multirow[t]{2}{*}{$\lambda_{8}$} & GF & 798.312 & 888.664 & 794.551 & 881.105 & 970.627 & 1063.2 & 1159.11 & 1257.52 \\
\hline & Ref. [1] & 798.6 & 888.6 & 794.7 & 880.3 & 968.5 & 1061.0 & 1158.7 & - \\
\hline \multirow{2}{*}{$\lambda_{9}$} & GF & 985.823 & 1086.19 & 981.983 & 1078.4 & 1178.33 & 1278.9 & 1373.23 & 1471.19 \\
\hline & Ref. [1] & 986.0 & 1086.0 & 981.6 & 1076.0 & 1175.0 & 1277.0 & 1384.0 & - \\
\hline
\end{tabular}


K.K. Żur

Table 4

The first ten lower dimensionless frequencies $\lambda$ of free vibration of simply supported circular plates

\begin{tabular}{|c|c|c|c|c|c|c|c|c|c|}
\hline \multirow{2}{*}{\multicolumn{2}{|c|}{$\begin{array}{c}\text { Dimensionless } \\
\text { frequency, } \lambda\end{array}$}} & \multicolumn{8}{|c|}{ The number of nodal diameters, $n$} \\
\hline & & 0 & 1 & 2 & 3 & 4 & 5 & 6 & 7 \\
\hline \multirow[t]{5}{*}{$\lambda_{0}$} & GF & 4.935 & 13.898 & 25.613 & 39.957 & 56.841 & 76.203 & 97.944 & 122.179 \\
\hline & Ref. [6] & 4.935 & 13.898 & 25.613 & 39.957 & 56.842 & 76.203 & - & - \\
\hline & Ref. [9] & 4.935 & 13.898 & 25.613 & 39.957 & 56.841 & 76.203 & - & - \\
\hline & Ref. [10] & 4.935 & 13.898 & 25.614 & 39.957 & 56.842 & 76.203 & - & - \\
\hline & Ref. [16] & 4.935 & 13.898 & 25.613 & - & - & - & - & - \\
\hline \multirow{5}{*}{$\lambda_{1}$} & GF & 29.72 & 48.478 & 70.117 & 94.549 & 121.702 & 151.518 & 183.948 & 218.951 \\
\hline & Ref. [6] & 29.720 & 48.479 & 70.117 & 94.549 & 121.702 & 151.518 & - & - \\
\hline & Ref. [9] & 29.72 & 48.478 & 70.117 & 94.549 & 121.702 & 151.518 & - & - \\
\hline & Ref. [10] & 29.71 & 48.478 & 70.116 & 94.549 & 121.702 & 151.518 & - & - \\
\hline & Ref. [16] & 29.72 & 8.478 & 70.117 & - & - & - & - & - \\
\hline \multirow[t]{5}{*}{$\lambda_{2}$} & $\mathrm{GF}$ & 74.156 & 102.773 & 134.298 & 168.675 & 205.851 & 245.778 & 88.414 & 33.721 \\
\hline & Ref. [6] & 74.156 & 102.772 & 134.298 & 168.675 & 205.851 & 245.778 & - & - \\
\hline & Ref. [9] & 74.156 & 102.773 & & 168.675 & 205.851 & 245.778 & - & - \\
\hline & Ref. [10] & 74.15 & 102.773 & 134.297 & 168.674 & 205.852 & 245.778 & - & - \\
\hline & Ref. [16] & 74.155 & 102.773 & 134.297 & - & - & - & - & - \\
\hline \multirow[t]{5}{*}{$\lambda_{3}$} & $\mathrm{GF}$ & 138.318 & 176.801 & 2 & 262.485 & 309.607 & 359.532 & 412.221 & 467.644 \\
\hline & Ref. [6] & 138.318 & 176.801 & 218.203 & 262.485 & 309.607 & 359.532 & - & - \\
\hline & Ref. [9] & 138.318 & 176.801 & 218.202 & 262.484 & 309.607 & 359.532 & - & - \\
\hline & Ref. [10] & 138320 & 176.801 & & 262.485 & 309.607 & 359.535 & - & - \\
\hline & Ref. [16] & 138. & 176.800 & 02 & - & - & - & - & - \\
\hline \multirow[t]{5}{*}{$\lambda_{4}$} & $\mathrm{GF}$ & 222.215 & 270.566 & 321.841 & 376.012 & 433.049 & 492.919 & 555.592 & 621.04 \\
\hline & Ref. [6 & 5 & & & 376.012 & 433.049 & 492.919 & 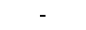 & . \\
\hline & Ref. [9] & 222.215 & 270.566 & 321. & 376.012 & 433.049 & 492.919 & - & - \\
\hline & Ref. [10] & 222.215 & 270.567 & 321.841 & 376.012 & 433.048 & 492.918 & - & - \\
\hline & Ref. [16] & 222.213 & 270.564 & & - & - & - & 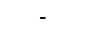 & 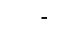 \\
\hline \multirow[t]{2}{*}{$\lambda_{5}$} & $\mathrm{GF}$ & 325.849 & 384.069 & 445.215 & 509.268 & 576.203 & 645.992 & 18.611 & 794.034 \\
\hline & Ref. [6] & 325.849 & 384.069 & 445.216 & 509.268 & 576.203 & 645.992 & - & - \\
\hline$\lambda_{6}$ & GF & 449.222 & 517.31 & 588.328 & 662.258 & 739.081 & 818.774 & 901.312 & 986.672 \\
\hline$\lambda_{7}$ & $\mathrm{GF}$ & 592.333 & 670.29 & & 834.984 & 921.690 & 1011.28 & 1103.81 & 1199.27 \\
\hline$\lambda_{8}$ & GF & 755.182 & 843.008 & 933.744 & 1027.46 & 1123.98 & 1223.59 & 1324.62 & 1427.13 \\
\hline$\lambda_{9}$ & GF & 937.764 & 1035.54 & 1136.62 & 1238.65 & 1346.82 & 1450.60 & 1560.95 & 1742.34 \\
\hline
\end{tabular}

Table 5

The first ten lower dimensionless frequencies $\lambda$ of free vibration of circular plates with sliding edge

\begin{tabular}{|c|c|c|c|c|c|c|c|c|c|}
\hline \multirow{2}{*}{\multicolumn{2}{|c|}{$\begin{array}{c}\text { Dimensionless } \\
\text { frequency, } \lambda\end{array}$}} & \multicolumn{8}{|c|}{ The number of nodal diameters, $n$} \\
\hline & & 0 & 1 & 2 & 3 & 4 & 5 & 6 & 7 \\
\hline \multirow[t]{3}{*}{$\lambda_{0}$} & GF & 14.682 & 3.082 & 8.784 & 16.902 & 27.343 & 40.055 & 55.003 & 72.160 \\
\hline & Ref. [6] & 14.682 & 3.082 & 8.785 & 16.902 & 27.343 & 40.056 & - & - \\
\hline & Ref.[15] & 14.682 & - & 8.785 & - & - & - & - & - \\
\hline \multirow[t]{3}{*}{$\lambda_{1}$} & $\mathrm{GF}$ & 49.218 & 28.398 & 44.904 & 64.130 & 86.004 & 110.464 & 137.462 & 166.958 \\
\hline & Ref. [6] & 49.218 & 28.399 & 44.904 & 64.130 & 86.004 & 110.464 & - & . \\
\hline & Ref. [15] & 49.218 & 28.399 & 44.904 & - & - & - & - & - \\
\hline \multirow[t]{3}{*}{$\lambda_{2}$} & $\mathrm{GF}$ & 103.499 & 72.859 & 99.361 & 128.677 & 160.754 & 195.539 & 232.990 & 273.067 \\
\hline & Ref. [6] & 103.499 & 72.859 & 99.361 & 128.677 & 160.754 & 195.539 & - & - \\
\hline & Ref. [15] & 103.500 & 72.860 & 99.359 & - & - & - & - & - \\
\hline \multirow[t]{3}{*}{$\lambda_{3}$} & GF & 177.521 & 137.025 & 173.442 & 212.716 & 254.806 & 299.671 & 347.273 & 397.579 \\
\hline & Ref. [6] & 177.521 & 137.025 & 173.442 & 212.716 & 254.806 & 299.671 & - & - \\
\hline & Ref. [15] & - & 137.009 & 173.564 & - & - & - & - & - \\
\hline \multirow{2}{*}{$\lambda_{4}$} & GF & 271.282 & 220.923 & 267.231 & 316.419 & 368.456 & 423.308 & 480.944 & 541.333 \\
\hline & Ref. [6] & 271.282 & 220.923 & 267.231 & 316.419 & 368.456 & 423.308 & - & - \\
\hline \multirow[t]{2}{*}{$\lambda_{5}$} & GF & 384.782 & 324.577 & 380.746 & 439.830 & 501.783 & 566.578 & 634.188 & 704.587 \\
\hline & Ref. [6] & - & 324.577 & 380.746 & 439.830 & 501.783 & 566.578 & - & - \\
\hline$\lambda_{6}$ & GF & 518.021 & 447.929 & 513.995 & 582.965 & 654.818 & 729.532 & 807.082 & 887.445 \\
\hline$\lambda_{7}$ & GF & 671.0 & 591.039 & 666.980 & 745.830 & 827.573 & 912.191 & 999.671 & 1090.01 \\
\hline$\lambda_{8}$ & GF & 843.718 & 753.888 & 839.699 & 928.440 & 1020.12 & 1114.57 & 1212.05 & 1312.14 \\
\hline$\lambda_{9}$ & GF & 1036.21 & 936.488 & 1032.24 & 1130.51 & 1231.13 & 1338.58 & 1435.74 & 1534.41 \\
\hline
\end{tabular}

Table 6

The first ten lower dimensionless frequencies $\lambda$ of free vibration of circular plates with elastic supports $(\varphi=0.1 ; \psi=100)$

\begin{tabular}{|c|c|c|c|c|c|c|c|c|c|}
\hline \multirow{2}{*}{\multicolumn{2}{|c|}{$\begin{array}{c}\text { Dimensionless } \\
\text { frequency, } \lambda\end{array}$}} & \multicolumn{8}{|c|}{ The number of nodal diameters, $n$} \\
\hline & & 0 & 1 & 2 & 3 & 4 & 5 & 6 & 7 \\
\hline \multirow[t]{3}{*}{$\lambda_{0}$} & GF & 4.854 & 12.139 & 19.079 & 25.685 & 33.427 & 43.294 & 55.625 & 70.451 \\
\hline & Ref. & 4.854 & 12.140 & 19.080 & 25.686 & 33.427 & 43.295 & - & - \\
\hline & Ref.[] & 4.854 & & & . & 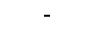 & 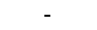 & - & - \\
\hline \multirow[t]{3}{*}{$\lambda_{1}$} & GF & 22.097 & & & 57.965 & 77.227 & 99.656 & 124.95 & 152.939 \\
\hline & Ref. [6 & & & & 57.965 & 77.227 & 99.657 & - & - \\
\hline & & & & & - & - & - & - & - \\
\hline \multirow[t]{3}{*}{$\lambda_{2}$} & & & & & 114.1 & 144.2 & 177.279 & 13.124 & 251.717 \\
\hline & & & & & 114.19 & 144.2 & 177.279 & - & - \\
\hline & Ref. & & & 85 & - & - & - & - & - \\
\hline \multirow[t]{2}{*}{$\lambda_{3}$} & GF & 9 & & 12 & 2.032 & 32.184 & 275.267 & 21.207 & 9.944 \\
\hline & Ref. [6 & & & & & 232.1 & 275.267 & - & - \\
\hline \multirow{2}{*}{$\lambda_{4}$} & GF & 9 & 27 & 102 & 290.168 & 340. & 393.257 & 449.155 & 507.890 \\
\hline & Ref. [6 & & & & 290.168 & 340.243 & 393.257 & - & - \\
\hline \multirow[t]{2}{*}{$\lambda_{5}$} & & & & & & & 531.121 & 6.924 & 5.594 \\
\hline & Ref. [6 & & & & & & & - & - \\
\hline$\lambda_{6}$ & GF & & & & & & 688.8 & 4.4 & 30 \\
\hline$\lambda_{7}$ & GF & & & & & 783.653 & 866.277 & 951.818 & 1040.36 \\
\hline ? & GF & 0 & 711.5 & 795.003 & 881.537 & 971.030 & 1063.60 & 1159.48 & 1255.58 \\
\hline$\lambda$ & GF & 798.765 & 889.108 & 982.443 & 1078.60 & 1178.52 & 1278.32 & 1373.61 & 1799.22 \\
\hline
\end{tabular}


Green's function approach to frequency analysis of thin circular plates

Table 7

The first ten lower dimensionless frequencies $\lambda$ of free vibration of circular plates with elastic supports $(\varphi=10 ; \psi=100)$

\begin{tabular}{cccccccccc}
\hline \hline \multirow{2}{*}{$\begin{array}{c}\text { Dimensionless } \\
\text { frequency, } \lambda\end{array}$} & \multicolumn{8}{c}{ The number of nodal diameters, $n$} \\
\cline { 2 - 11 }$\lambda_{0}$ & GF & 7.790 & 13.845 & 19.352 & 25.740 & 34.090 & 44.808 & 57.962 & 73.504 \\
& Ref. [6] & 7.790 & 13.845 & 19.352 & 25.741 & 34.090 & 44.809 & - & - \\
& Ref.[15] & 7.790 & 13.845 & 19.352 & - & - & - & - & - \\
$\lambda_{1}$ & GF & 22.128 & 32.098 & 45.801 & 63.015 & 83.282 & 106.348 & 132.075 & 160.377 \\
& Ref. [6] & 22.128 & 32.098 & 45.802 & 63.016 & 83.282 & 106.348 & - & - \\
& Ref. [15] & 22.128 & 32.098 & 45.802 & - & - & - & - & - \\
$\lambda_{2}$ & GF & 49.253 & 70.468 & 95.064 & 122.728 & 153.294 & 186.663 & 222.764 & 261.542 \\
& Ref. [6] & 49.254 & 70.468 & 95.065 & 122.728 & 153.294 & 186.663 & - & - \\
& Ref. [15] & 49.254 & 70.468 & 95.064 & - & - & - & - & - \\
$\lambda_{3}$ & GF & 98.741 & 130.265 & 164.874 & 202.466 & 242.962 & 286.302 & 332.436 & 381.320 \\
& Ref. [6] & 98.741 & 130.265 & 164.874 & 202.466 & 242.962 & 286.302 & - & - \\
$\lambda_{4}$ & GF & 168.599 & 210.017 & 254.463 & 301.883 & 352.226 & 405.447 & 461.505 & 520.364 \\
& Ref. [6] & 168.599 & 210.017 & 254.463 & 301.883 & 352.226 & 405.447 & - & - \\
$\lambda_{5}$ & GF & 258.213 & 309.453 & 363.707 & 420.938 & 481.108 & 544.179 & 610.119 & 678.895 \\
& Ref. [6] & 258.213 & 309.453 & 363.707 & 420.938 & 481.108 & 544.180 & - & - \\
$\lambda_{6}$ & GF & 367.477 & 428.531 & 492.594 & 559.638 & 629.632 & 702.545 & 778.349 & 857.013 \\
$\lambda_{7}$ & GF & 496.382 & 567.255 & 641.135 & 717.998 & 797.818 & 880.574 & 966.209 & 1054.78 \\
$\lambda_{8}$ & GF & 644.938 & 725.639 & 809.342 & 896.037 & 985.712 & 1078.17 & 1174.70 & 1272.15 \\
$\lambda_{9}$ & GF & 813.16 & 903.712 & 997.277 & 1093.80 & 1193.01 & 1300.43 & 1379.91 & 1497.66 \\
\hline
\end{tabular}

Table 8

The first ten lower dimensionless frequencies $\lambda$ of free vibration of circular plates with elastic supports $(\varphi=1000 ; \psi=100)$

\begin{tabular}{|c|c|c|c|c|c|c|c|c|c|}
\hline \multirow{2}{*}{\multicolumn{2}{|c|}{$\begin{array}{c}\text { Dimensionless } \\
\text { frequency, } \lambda\end{array}$}} & \multicolumn{8}{|c|}{ The number of nodal diameters, $n$} \\
\hline & & 0 & 1 & 2 & 3 & 4 & 5 & 6 & 7 \\
\hline \multirow[t]{3}{*}{$\lambda_{0}$} & GF & 8.809 & 14.552 & 19.475 & 25.769 & 34.480 & 45.828 & 59.746 & 76.123 \\
\hline & Ref. [6] & 8.809 & 14.552 & 19.475 & 25.769 & 34.481 & 45.828 & - & - \\
\hline & Ref.[15] & 8.809 & 14.552 & 19.475 & - & - & - & - & - \\
\hline \multirow[t]{3}{*}{$\lambda_{1}$} & GF & 22.142 & 32.628 & 47.554 & 65.992 & 87.405 & 111.561 & 138.339 & 167.666 \\
\hline & Ref. [6] & 22.143 & 32.629 & 47.554 & 65.993 & 87.406 & 111.561 & - & - \\
\hline & Ref. [15] & 22.142 & 32.629 & 47.554 & - & - & - & - & - \\
\hline \multirow[t]{3}{*}{$\lambda_{2}$} & GF & 51.441 & 74.280 & 100.364 & 129.418 & 161.309 & 195.953 & 233.288 & 273.267 \\
\hline & Ref. [6] & 51.442 & 74.281 & 100.364 & 129.418 & 161.309 & 195.953 & - & - \\
\hline & Ref. [15] & 51.442 & 74.280 & 100.366 & - & - & - & - & - \\
\hline \multirow[t]{2}{*}{$\lambda_{3}$} & GF & 104.413 & 137.654 & 173.880 & 213.012 & 254.988 & 299.755 & 347.270 & 397.496 \\
\hline & Ref. [6] & 104.413 & 137.654 & 173.880 & 213.012 & 254.988 & 299.755 & - & - \\
\hline \multirow[t]{2}{*}{$\lambda_{4}$} & GF & 177.926 & 221.172 & 267.357 & 316.442 & 368.387 & 423.155 & 480.711 & 541.024 \\
\hline & Ref. [6] & 177.926 & 221.172 & 267.357 & 316.442 & 368.387 & 423.155 & - & - \\
\hline \multirow[t]{2}{*}{$\lambda_{5}$} & GF & 271.391 & 324.553 & 380.643 & 439.636 & 501.504 & 566.217 & 633.747 & 704.066 \\
\hline & Ref. [6] & 271.391 & 324.553 & 380.643 & 439.636 & 501.504 & 566.217 & - & - \\
\hline$\lambda_{6}$ & GF & 384.668 & 447.716 & 513.690 & 582.572 & 654.339 & 728.968 & 806.434 & 886.713 \\
\hline$\lambda_{7}$ & GF & 517.708 & 590.630 & 666.479 & 745.238 & 826.891 & 911.421 & 998.782 & 1089.06 \\
\hline$\lambda_{8}$ & GF & 670.492 & 753.282 & 838.999 & 927.644 & 1019.22 & 1113.55 & 1211.74 & 1310.34 \\
\hline$\lambda_{9}$ & GF & 843.007 & 935.670 & 1031.21 & 1129.53 & 1229.99 & 1337.24 & 1424.49 & 1540.84 \\
\hline
\end{tabular}

\section{Conclusions}

In this paper, the Green's functions have been employed to solve natural vibration of circular thin plates with different boundary conditions. The universal Green's function for different nodal diameters is defined in a closed form. The ten lower natural frequencies are calculated for eight different natural modes. The limited independent solutions of differential Euler equations were expanded into the Neumann power series using the method of successive approximation. This approach allows to obtain the analytical frequency equations as power series rapidly convergent to exact eigenvalues for a different number of nodal diameters. The obtained results are in good agreement with results obtained by other methods presented in literature. The obtained numerical results can be used to validate the accuracy of other numerical methods as benchmark values. The calculations are evaluated with the help of Mathematica v10, which is a symbolic calculation software.

\section{REFERENCES}

[1] A.W. Leissa, Vibration of Plates, National Aeronautics and Space Administration, Washington, 1969.

[2] H.F. Bauer and W. Eidel, "Determination of the lower natural frequencies of circular plates with mixed boundary conditions", J. Sound and Vibration 292, 742-764 (2006).

[3] S. Chakraverty and M. Petyt, "Natural frequencies for free vibration of nonhomogeneous elliptic and circular plates using two-dimensional orthogonal polynomials", Applied Mathematical Modelling 21 (7), 399-417 (1997).

[4] R.B. Bhat, S. Chakraverty, and I. Stiharu, "Recurrence scheme for the generation of two-dimensional boundary characteristic orthogonal polynomials to study vibration of plates", J. Sound and Vibration 216 (2), 321-327 (1998).

[5] T.Y. Wu and G.R. Liu, "Free vibration analysis of circular plates with variable thickness by the generalized differential quadrature rule", Int. J. Solids and Structures 38, 7967-7980 (2001).

[6] T.Y. Wu, Y.Y. Wang, and G.R. Liu, "Free vibration analysis of circular plates using generalized differential quadrature rule", 
Computer Methods in Applied Mechanics and Engineering 191, 5365-5380 (2002).

[7] J. Jaroszewicz and L. Zoryj, "The method of partial discretization in free vibration problems of circular plates with variable distribution of parameters", Int. Applied Mechanics 42 (3), 364-373 (2006).

[8] F. Ebrahimi and A. Rastgo, "An analytical study on the free vibration of smart circular thin FGM plate based on classical plate theory", Thin-Walled Structures 46 (12), 1402-1408 (2008).

[9] H.S. Yalcin, A. Arikoglu, and I. Ozkol, "Free vibration analysis of circular plates by differential transformation method", Applied Mathematics and Computation 212, 377-386 (2009).

[10] Z.H. Zhou, K.W. Wong, X.S. Xu, and A.Y.T. Leung, "Natural vibration of circular and annular thin plates by Hamiltonian approach", J. Sound and Vibration 330, 1005-1017 (2011).

[11] S. Kukla and M. Szewczyk, "Frequency analysis of annular plates with elastic concentric supports by Green's function method", J. Sound and Vibration 300, 387-393 (2007).
[12] S.V. Sorokin and N. Peake, "Vibrations of sandwich plates with concentrated masses and spring - like inclusions", J. Sound and Vibration 237 (2), 203-222 (2000).

[13] S. Kukla, Green's Functions and Their Properties, Czestochowa University of Technology, Czestochowa, 2009.

[14] J. Jaroszewicz and L. Zoryj, Methods of Free Axisymmetric Vibration Analysis of Circular Plates Using by Influence Functions, Bialystok University of Technology, Bialystok, 2005.

[15] S. Azimi, "Free vibration of circular plates with elastic edge supports using receptance method", J. Sound and Vibration 120, 37-52 (1988)

[16] G. Duan, X. Wang, and Ch. Jin, "Free vibration analysis of circular thin plates with stepped thickness by the DSC element method", Thin-Walled Structures 85, 25-33 (2014).

[17] F.G. Tricomi, Integral Equations, Dover Publications, New York, 1985

[18] Y.V. Shestopalov and Y.G. Smirnov, Integral Equations, Karlstad University, Karlstad, 2002. 